\title{
A MEAN ERGODIC THEOREM
}

\section{ROBERT SINE}

Let $T$ be a bounded linear operator on a Banach space $B$. We will call $T$ norm mean stable if the Cesaro means $A_{N}(T)=(N+1)^{-1} \sum_{0}^{N} T^{k}$ converge in the strong operator topology. We prove the following:

THEOREM. Let $T$ be a contraction. Then $T$ is norm mean stable if and only if the fixed points of $T$ separate the fixed points of $T^{*}$.

Our first lemma summarizes known results in spectral theory. Only item (d) requires $\|T\| \leqq 1$; this fact follows from the KakutaniMarkov fixed point theorem.

Lemma. Let $T$ be a contraction on $B$. Then

(a) 1 is in $\sigma(T)$ iff it is in the spectrum of $T^{*}$.

(b) If 1 is in the point spectrum of $T^{*}$ it is in either the point spectrum or the residual spectrum of $T$.

(c) If 1 is in the residual spectrum of $T$ it is in the point spectrum of $T^{*}$.

(d) If 1 is in the point spectrum of $T$ it is in the point spectrum of $T^{*}$.

Our next lemma is a special case of the norm ergodic theorem [ 1 , p. 661]. We include a proof for completeness.

Lemma. Let $\|T\| \leqq 1$. Then $A_{N}(T)$ converges in the weak operator topology iff it converges in the strong operator topology.

Proof. That strong implies weak needs no comment. So we choose $x$ in the range of $I-T$. Then $A_{N}(T) x=A_{N}(T)(I-T) w$ $=N^{-1}\left(T-T^{N+1}\right) w \rightarrow 0$. This clearly holds for the closure of the range as well by approximation. Now the projection which is the weak operator limit can be employed to reduce the problem to the case that $A_{N}(T) \rightarrow 0$ in the weak operator topology. In this case, however, $I-T$ is necessarily either onto or has dense range since $P^{*}$, and therefore $T^{*}$, has no fixed points.

Proof of Theorem 1. First we suppose $A_{N}(T) x$ converges in norm for all $x$ in $B$. If $x_{1}^{*} \neq x_{2}^{*}$ are invariant choose any vector $x$ with $\left(x, x_{1}^{*}\right) \neq\left(x, x_{2}^{*}\right)$. Let $\bar{x}$ be the limit of $A_{N}(T) x$. Now $\left(x, x^{*}\right)=\left(\bar{x}, x^{*}\right)$ whenever $x^{*}$ is invariant.

Received by the editors May 20, 1968.

1 This research was partially supported by the National Science Foundation. 
Now we suppose the separation condition holds. Any cluster point of $A_{N}\left(T^{*}\right) x^{*}$ is invariant under $T^{*}$; compactness of the unit ball of $B^{*}$ guarantees at least one cluster point and the separation condition guarantees at most one. Thus we have convergence of $A_{N}\left(T^{*}\right) x^{*}$ in the $\omega^{*}$ topology. Let $\phi$ denote the map of the unit ball of $B^{*}$ defined by $\phi\left(x^{*}\right)=\bar{x}^{*}=\lim A_{N}\left(T^{*}\right) x^{*}$. If $x^{*}(\alpha)$ converges to $x^{*}(0)$ then for each invariant vector $x$ we have $\left(x, x^{*}(\alpha)\right) \rightarrow\left(x, x^{*}(0)\right)$. Thus $\phi$ is continuous with the $\omega^{*}$ topology on the domain and the topology on the range space that is generated by the invariant vectors regarded as functionals on $B^{*}$. But the range $\phi$ is $\omega^{*}$ compact since the subspace of $T^{*}$ fixed points is $\omega^{*}$ closed. The topology generated by the invariant vectors is Hausdorff by separability and is formally weaker than the $\omega^{*}$ topology; therefore these topologies are identical. Now given $x$ in $B$ define the functional $f\left(x^{*}\right)=\left(x, \bar{x}^{*}\right)$. Since $f$ is continuous in the bounded $\omega^{*}$ topology it is $\omega^{*}$ continuous $[1$, p. 428]. Then $x \rightarrow \bar{x}=f$ defines a projection operator on $B$ which is the weak operator limit of $A_{N}(T)$. The previous theorem now completes the proof.

Remark. The convergence of $A_{N}\left(T^{*}\right) x^{*}$ in the $\omega^{*}$ topology does not imply weak operator convergence of $A_{N}(T)$. Consider on $C[0,1]$ the operator $T f(x)=f\left(x^{2}\right)$. We have dominated pointwise convergence for $T^{k} f$ but the limit function is not in general a continuous function so that $A_{N}\left(T^{*}\right) x^{*}$ converges in the $\omega^{*}$ topology but $A_{N}(T)$, while weakly Cauchy, does not converge in the weak operator topology.

It should be mentioned that separation of the extremes of the set of invariant measures of norm less than or equal to one is not sufficient for norm mean stability. See [2] for a counterexample. The relation between this separation of extremes and norm mean stability will be discussed in a later paper. This result was previously established for Markov operators acting on continuous functions on a compact Hausdorff space [2].

\section{REFERENCES}

1. N. Dunford and J. Schwartz, Linear operators. I: General theory, Pure and Appl. Math., vol. 7, Interscience, New York, 1958. MR 22 \#8302.

2. R. Sine, A geometric theory for a single Markov operator, Pacific J. Math. 27 (1969), 155-166.

University of Minnesota AND

Control Data Corporation 